\title{
Deficient antigen-specific T cell responses in SLE-prone mice
}

Systemic lupus erythematosus (SLE) is associated with increased susceptibility to infections, a major cause of morbidity and mortality in patients with this disease. In a study published in PLOS ONE, Lieberman and Tsokos show that lupus-prone mice have lower antigen-specific T cell responses to intracellular pathogensa possible explanation for the increased susceptibility to infection observed in patients with SLE.

\section{4 ...revealing a T cell-intrinsic defect in immune function in lupus-prone mice 77}

Finding the reasons for the increased rates of infection observed in patients with SLE is complicated by both widespread immune dysregulation and by the multiple effects of immunosuppressive therapies in these patients. To circumvent these confounding factors, researchers used two genetically diverse strains of lupus-prone mice (B6.lpr and BXSB) infected with Toxoplasma gondii prior to development of active disease, and compared their immune response with that of wild-type mice.

Both strains of lupus-prone mice had higher parasite counts and increased mortality following T. gondii infection when compared with wild-type mice. Intriguingly, serum levels of interferon (IFN) $-\gamma$ measured at the peak of infection were reduced in BXSB mice (although not in B6.lpr mice) than wild-type controls $(P<0.0001)$. Moreover, whereas T-cellactivation markers were similar in infected lupus-prone and wild-type mice, purified splenocytes recovered from infected lupusprone mice showed lower production of IFN- $\gamma$ in response to $T$. gondii-specific antigen when compared with infected wild-type controls. Importantly, this difference in IFN- $\gamma$ production was not observed following polyclonal stimulation.

To understand this functional deficiency further, T cells and dendritic cells were purified from either lupus-prone or wildtype infected mice, co-cultured in the presence of antigen-specific stimulation and assessed for production of IFN- $\gamma$. In the presence of dendritic cells from either lupus-prone or wild-type mice, $\mathrm{T}$ cells from both B6.lpr and BXSB mice showed significantly lower IFN- $\gamma$ production than wild-type T cells $(P=0.0064$ and $P=0.0008$, respectively), revealing a T cellintrinsic defect in immune function in lupus-prone mice.

Whether patients with SLE also have $\mathrm{T}$ cell-intrinsic functional deficiencies remains to be determined. Nevertheless, these findings provide a fresh perspective on the mechanisms causing increased susceptibility to infection in patients with systemic autoimmune diseases, and might help shape therapeutic approaches aimed at reducing infection-related morbidity and mortality in these patients.

João H. Duarte

Original article Lieberman, L. A. and Tsokos, G. C. Lupusprone mice fail to raise antigen-specific $T$ cell responses to intracellular infection. PLOS ONE 9, e111382 (2014) 